\title{
Enhanced Modal Approach for Free-flight Nonlinear Aeroelastic Simulation of Very Flexible Aircraft
}

\author{
Markus Ritter* \\ DLR - Institute of Aeroelasticity, Bunsenstraße 10, 37073 Göttingen, Germany \\ Jessica Jones ${ }^{\dagger}$ \\ University of Michigan, Ann Arbor, Michigan 48109-2140 \\ Carlos E. S. Cesnik $\ddagger$ \\ University of Michigan, Ann Arbor, Michigan 48109-2140
}

\begin{abstract}
A recently proposed modal-based method that captures the geometric nonlinear effects in the regime of large deformations is applied to the modeling of University of Michigan's $\mathrm{X}$-HALE UAV. The method is an extension of the classical modal approach towards large geometric deflections and uses higher-order stiffness terms and mode components to account for nonlinear force-displacement relationships and geometrically nonlinear displacement fields. In this paper, the method is further extended to enable rigid body motions of an aircraft in free flight. The verification of this extension is done using the X-HALE model which is considered as a challenging test case due to pronounced nonlinearities in steady and unsteady flight conditions. A structural and an aerodynamic model of the X-HALE UAV were built and aeroelastic simulations of the model were done and validated with UM/NAST results.
\end{abstract}

\section{Introduction}

7 HE recently developed extension of the classical modal approach towards large deformations (refered to 1 as Enhanced Modal Approach) allows the modeling of large structural deflections based on higher-order mode components. ${ }^{1}$ This approach uses a higher order strain energy formulation to consider nonlinear forcedisplacement relationships and higher-order mode components for the reconstruction of a geometrically nonlinear displacement field. Both enhancements are derived by Taylor series expansions; a number of static, nonlinear structural simulations of the structure considered is necessary to identify the higher-order components.

One of the consequential extensions of the enhanced modal approach is the ability to model highly flexible free-flying aircraft. This requires the derivation of governing equations that account for the vehicle's rigid body motion. Especially the coupling between flight dynamic and elastic degrees of freedom must be accounted for since low-frequency structural modes interact heavily with rigid body flight dynamic modes and large deflections render the aerodynamic and inertia properties of the vehicle strongly state-dependent. ${ }^{2}$ An existing aeroelastic toolbox used for the unsteady analysis of elastic aircraft in the time domain at the German Aerospace Center (DLR) was extended by the enhanced modal approach. Therefore, the governing equations which include all necessary coupling terms between rigid-body and elastic degrees of freedom (inertial coupling) were derived. Aerodynamic forces are calculated by an unsteady vortex-lattice method, the transfer of forces and deformations between the aerodynamic and structural part is done via a coupling approach that was successfully used for large deformations. ${ }^{1}$ An implicit numerical scheme is used to advance the solution of the coupled system in time.

Besides the implementation of the simulation framework, a representative test case must be found for its verification. The X-HALE UAV from University of Michigan's Active Aeroelasticity and Structures Research

\footnotetext{
*Research Engineer, AIAA Member (markus.ritter@dlr.de)

${ }^{\dagger}$ Graduate Research Assistant, Department of Aerospace Engineering (jrenejo@umich.edu)

‡Professor, Department of Aerospace Engineering, Fellow, AIAA (cesnik@umich.edu)
} 
Laboratory is considered as a perfect test case for this purpose. The concept of the model is shown in Fig. 1. A range of simulation results from the UM/NAST toolbox is available for this configuration to support the

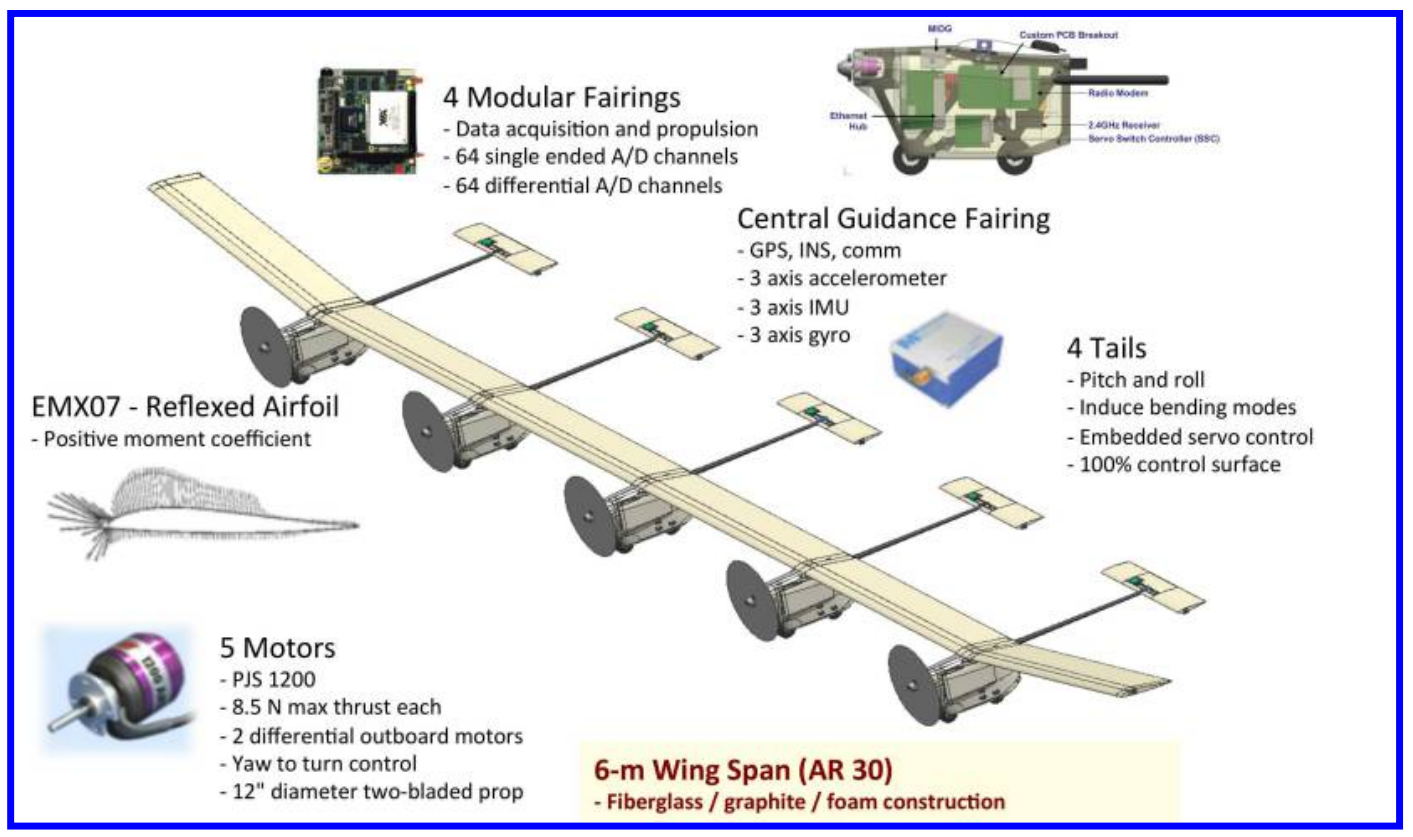

Figure 1. Concept of the X-HALE UAV for nonlinear aeroelastic tests developed and build by University of Michigan. ${ }^{3}$

numerical verification and validation of the enhanced modal approach applied to the free-flying aircraft.

Prior and recent activities of researchers working on the reduced order modeling (ROM) of nonlinear structures are explained shortly in order to integrate the enhanced modal approach within this field.

Mignolet and co-workers have given detailed descriptions for the derivation of static and dynamic nonlinear structural governing equations including quadratic and cubic stiffness coefficients. The field of application ranges from plate structures to curved beams and the modeling of a complex UAV wing. ${ }^{4,5,6}$

Kuether and Allen describe methods based on nonlinear normal modes (NNMs) to simplify large, complex structures. NNMs basically describe the resonant frequency and response of a structure as a function of response amplitude or energy. ${ }^{7,8}$ Quadratic and cubic stiffness terms are used to consider nonlinear force displacement behaviour and coupling of individual modes.

As will be shown, the kinematically nonlinear displacement field present in large deformations of wing-like structures can be reconstructed by shape functions of higher order. The method of quadratic components was successfully applied by Segalman and Dohrmann to improve the kinematical and dynamical description of rotating structures (beams) undergoing large displacements. ${ }^{9,10}$ This method is also used by van Zyl for the calculation of T-Tail flutter. ${ }^{11,12}$

This paper describes the derivation of the enhanced modal approach in a compact form. The setup of a structural, an aerodynamic, and a coupling model of the X-HALE aircraft are described in detail. Results of static structural simulations, steady aeroelastic (trimming), and unsteady manoeuvre simulations are presented and compared to results of UM/NAST.

\section{Derivation of the Enhanced Modal Approach}

The derivation of the enhanced modal approach is given in detail in Ref. $^{1}$ and recapitulated in a more compact form in the following.

Compared to the classical modal approach, the proposed method is based upon considering a nonlinear force-displacement relationship by quadratic and cubic stiffness terms and a geometrically nonlinear displacement field based on higher-order mode components up to fourth order. Furthermore, the generalized forces are dependent on the state of deformation and the nodal force field applied. 


\section{II.A. Static formulation: Higher-order strain energy}

The total potential energy of an elastic body $U$ consists of the sum of the total strain energy $U$ and the potential energy of the applied loads $V$. This statement is expressed as: ${ }^{13}$

$$
\pi=U+V .
$$

Assuming linear elastic material behaviour (Hooke's law), and neglecting the influence of temperature, the total strain energy $U$ in the body is given as (here and what follows, the Einstein notation is used):

$$
U=\frac{1}{2} \int_{V} \tau_{i j} \epsilon_{i j} d v(i, j=1,2,3),
$$

where $\tau_{i j}$ and $\epsilon_{i j}$ are the stress and strain component pairs, respectively. The work done by the applied loads $V$ can be expressed as:

$$
V=\int_{V} B_{i} u_{i} d v+\int_{S} T_{i}^{(\nu)} u_{i} d s,
$$

where $B_{i}$ represents the components of the applied body forces, $u_{i}$ the components of the displacement field, and $T_{i}^{(\nu)}$ denotes the traction vector applied on the body surface.

Introducing a variation of both the total strain energy and the external work yields:

$$
\delta \pi=\delta(U+V)=\int_{V} \tau_{i j} \delta \epsilon_{i j} d v-\int_{V} B_{i} \delta u_{i} d v-\int_{S} T_{i}^{(\nu)} \delta u_{i} d s .
$$

Invoking the Principle of Minimum Total Potential Energy, i.e., $\delta \pi=0$, yields the variation of the total strain energy equals the negative variation of the external work:

$$
\delta U=-\delta V .
$$

Now the total strain energy, Eq.(2), is assumed to be a nonlinear and continuously differentiable function of a scalar value $q_{i}$ of a number of generalized cordinates, $m$, and expanded in a Taylor series up to the fourth order centered at zero (since the internal energy is zero for zero deformation):

$$
\begin{aligned}
U(\boldsymbol{q}) & =\frac{1}{2 !} \sum_{i=1}^{m} \sum_{j=1}^{m} \frac{\partial^{2} U}{\partial q_{i} \partial q_{j}} q_{i} q_{j} \\
& +\frac{1}{3 !} \sum_{i=1}^{m} \sum_{j=1}^{m} \sum_{k=1}^{m} \frac{\partial^{3} U}{\partial q_{i} \partial q_{j} \partial q_{k}} q_{i} q_{j} q_{k} \\
& +\frac{1}{4 !} \sum_{i=1}^{m} \sum_{j=1}^{m} \sum_{k=1}^{m} \sum_{l=1}^{m} \frac{\partial^{4} U}{\partial q_{i} \partial q_{j} \partial q_{k} \partial q_{l}} q_{i} q_{j} q_{k} q_{l}+\text { h.o.t. }
\end{aligned}
$$

Using Castigliano's first theorem and applying a differentiation with respect to $\boldsymbol{q}$ centered at zero yields a governing equation of the structure as a function of $\boldsymbol{q} \cdot{ }^{13}$ This governing equation comprises quadratic and cubic stiffness dependencies and thus accounts for a nonlinear force-deformation behaviour:

$$
\begin{aligned}
\frac{\partial U}{\partial q_{p}} & =\sum_{i=1}^{m} \frac{\partial^{2} U}{\partial q_{i} \partial q_{p}} q_{i} \\
& +\frac{1}{2 !} \sum_{i=1}^{m} \sum_{j=1}^{m} \frac{\partial^{3} U}{\partial q_{i} \partial q_{j} \partial q_{p}} q_{i} q_{j} \\
& +\frac{1}{3 !} \sum_{i=1}^{m} \sum_{j=1}^{m} \sum_{k=1}^{m} \frac{\partial^{4} U}{\partial q_{i} \partial q_{j} \partial q_{k} \partial q_{p}} q_{i} q_{j} q_{k}+\text { h.o.t. }
\end{aligned}
$$

Introducing the definitions ${ }^{p} G_{n}$ for the partial derivatives in Eq.(7), the following equation in the pseudogeneralized coordinates is obtained and used as the basis for the method:

$$
{ }^{p} G_{1}^{i} q_{i}+{ }^{p} G_{2}^{i j} q_{i} q_{j}+{ }^{p} G_{3}^{i j k} q_{i} q_{j} q_{k}=Q^{p} \quad(p=1, \ldots, m)
$$


Here the summation convention is used again. The idea of the proposed method is to take the ${ }^{p} G_{n}$ stiffness matrices as generalized stiffnesses and the $\boldsymbol{q}$ as generalized coordinates. The stiffness parameters $G_{n}$ can be determined by polynomial fitting or numerical differentiation. ${ }^{1}$

\section{II.B. Static formulation: Higher-order deformation reconstruction}

The second extension is the reconstruction of the geometrically nonlinear displacement field. As mentioned above, the nonlinear static FE analysis excites nonlinear terms in the strain energy, but also a nonlinear displacement field.

In this work, the nodal deformation field is expanded in a Taylor series centered at zero (similar to the strain energy) which is truncated after the fourth term:

$$
\begin{aligned}
\mathbf{u}(\boldsymbol{q})= & \sum_{i=1}^{m} \frac{\partial \mathbf{u}}{\partial q_{i}} q_{i} \\
& +\frac{1}{2 !} \sum_{i=1}^{m} \sum_{j=1}^{m} \frac{\partial^{2} \mathbf{u}}{\partial q_{i} \partial q_{j}} q_{i} q_{j} \\
& +\frac{1}{3 !} \sum_{i=1}^{m} \sum_{j=1}^{m} \sum_{k=1}^{m} \frac{\partial^{3} \mathbf{u}}{\partial q_{i} \partial q_{j} \partial q_{k}} q_{i} q_{j} q_{k} \\
& +\frac{1}{4 !} \sum_{i=1}^{m} \sum_{j=1}^{m} \sum_{k=1}^{m} \sum_{l=1}^{m} \frac{\partial^{4} \mathbf{u}}{\partial q_{i} \partial q_{j} \partial q_{k} \partial q_{l}} q_{i} q_{j} q_{k} q_{l}+\text { h.o.t. }
\end{aligned}
$$

Partial differentiation of the displacement field defined in this way with respect to the generalized coordinates and substitution similar as above for the strain energy yields:

$$
{ }^{p} \boldsymbol{\Phi}=\frac{\partial \mathbf{u}}{\partial q_{p}}={ }^{p} \boldsymbol{\Phi}_{0}+{ }^{p} \boldsymbol{\Phi}_{1}^{i} q_{i}+{ }^{p} \boldsymbol{\Phi}_{2}^{i j} q_{i} q_{j}+{ }^{p} \boldsymbol{\Phi}_{3}^{i j k} q_{i} q_{j} q_{k}
$$

The term ${ }^{p} \boldsymbol{\Phi}_{0}$ can be seen as the equivalent of the structure's normal modes.

\section{II.B.1. The generalized forces and the final governing equation}

Considering Eq.(8), the forcing term of the structure's governing equation consists of the generalized force $Q^{p}$. Applying the principle of virtual work and the Taylor series expansion of the higher order mode components results in an extension of the generalized forces. For simplicity, the approach described by van Zyl is followed here and only the linear and the quadratic mode shape components are considered for the virtual work: ${ }^{12}$

$$
\delta V=\delta \mathbf{u}^{T} \boldsymbol{f},
$$

where $\boldsymbol{f}$ denotes an arbitrary nodal force field. Expansion of the virtual physical displacements $\delta \mathbf{u}$ using the linear and the quadratic mode shapes yields:

$$
\delta V=\delta q_{p}^{T}\left({ }^{p} \boldsymbol{\Phi}_{0}^{T}+{ }^{p} \boldsymbol{\Phi}_{1}^{i^{T}} q_{i}\right) \boldsymbol{f} .
$$

Thus the generalized forces are given as:

$$
Q^{p}={ }^{p} \boldsymbol{\Phi}_{0}^{T} \boldsymbol{f}+{ }^{p} \boldsymbol{\Phi}_{1}^{i^{T}} \boldsymbol{f} q_{i}
$$

Combining Eq.(8) and (16) yields:

$$
\left({ }^{p} G_{1}^{i}-{ }^{p} \boldsymbol{\Phi}_{1}^{i^{T}} \boldsymbol{f}\right) q_{i}+{ }^{p} G_{2}^{i j} q_{i} q_{j}+{ }^{p} G_{3}^{i j k} q_{i} q_{j} q_{k}={ }^{p} \boldsymbol{\Phi}_{0}{ }^{T} \boldsymbol{f} .
$$

Eq.(17) is the static governing equation of the enhanced modal approach. Compared to the classical modal approach, it can be seen that the ${ }^{p} G_{1}^{i}$ term is amended by the product of the transpose of the quadratic mode 
component matrix and the force field. This additional stiffness parameter is proportional to the force field applied to the structure and induces a coupling of the otherwise (in the linear sense) uncoupled eigenvectors.

Following the solution of Eq.(17), the nodal deformation field is reconstructed as function of the generalized coordinates $\boldsymbol{q}$ :

$$
\mathbf{u}(\boldsymbol{q})={ }^{p} \boldsymbol{\Phi}_{0} q_{p}+{ }^{p} \boldsymbol{\Phi}_{1}^{i} q_{p} q_{i}+{ }^{p} \boldsymbol{\Phi}_{2}^{i j} q_{p} q_{i} q_{j}+{ }^{p} \boldsymbol{\Phi}_{3}^{i j k} q_{p} q_{i} q_{j} q_{k}
$$

where the sum is taken again over repeated indices.

\section{II.C. Dynamic formulation}

The dynamic formulation is obtained by adding inertia terms to Eq.(17). The governing equation is then given as (neglecting velocity-dependent damping forces for simplicity):

$$
\boldsymbol{M} \ddot{q}_{i}+\left({ }^{p} G_{1}^{i}-{ }^{p} \boldsymbol{\Phi}_{1}^{i}{ }^{T} \boldsymbol{f}\right) q_{i}+{ }^{p} G_{2}^{i j} q_{i} q_{j}+{ }^{p} G_{3}^{i j k} q_{i} q_{j} q_{k}={ }^{p} \boldsymbol{\Phi}_{0}{ }^{T} \boldsymbol{f},
$$

with $M$ denoting the generalized mass matrix.

\section{II.D. Flight dynamic equations with elastic and rigid body degrees of freedom}

The governing equations of the unsteady simulations comprise both rigid body and elastic degrees of freedom. Lagrange's equations of the second kind are used to derive a set of coupled equations, where the coupling manifests between the rigid body and elastic degrees of freedom in off-diagonal terms in the mass and gyroscopic matrices. This also means that no mean-axis conditions are used to decouple rigid body and elastic degrees of freedom. ${ }^{14,15}$ A reason for this approach is that the calculation of the higher order stiffness and mode components by a series of static nonlinear solutions requires the structural model to be clamped in at least one node. No inertia relief exists so far to be used with large deformation simulations, thus the governing equations of the flexible aircraft are formulated such that clamped mode shapes can be used. Rigid body mode shapes could be used to simplify the solution process, but large pitch motions are to be expected during strong manoeuvres which cannot be simulated satisfactorily by rigid body modes.

Without further derivation, the final form of the combined first and second order nonlinear governing equations of the flexible aircraft is given in matrix form as:

$$
\left[\begin{array}{ll}
\boldsymbol{M}_{B B} & \boldsymbol{M}_{B E} \\
\boldsymbol{M}_{E B} & \boldsymbol{M}_{E E}
\end{array}\right]\left[\begin{array}{c}
\stackrel{\boldsymbol{\beta}}{ } \\
\ddot{\boldsymbol{q}}
\end{array}\right]+\left[\begin{array}{ll}
\boldsymbol{D}_{B B} & \boldsymbol{D}_{B E} \\
\boldsymbol{D}_{E B} & \boldsymbol{D}_{E E}
\end{array}\right]\left[\begin{array}{c}
\boldsymbol{\beta} \\
\dot{\boldsymbol{q}}
\end{array}\right]+\left[\begin{array}{cc}
\mathbf{0} & \mathbf{0} \\
\mathbf{0} & \boldsymbol{K}
\end{array}\right]\left[\begin{array}{l}
\mathbf{0} \\
\boldsymbol{q}
\end{array}\right]=\left[\begin{array}{c}
\tilde{\boldsymbol{Q}}_{B} \\
\boldsymbol{Q}_{E}
\end{array}\right] .
$$

The vector $\boldsymbol{\beta}$ of the independent variables of the body-fixed frame degrees of freedom is defined as follows:

$$
\boldsymbol{\beta}=\left[\begin{array}{l}
\boldsymbol{V}_{b} \\
\boldsymbol{\omega}_{b}
\end{array}\right]
$$

where $\boldsymbol{V}_{b}$ denotes the body frame's translational velocities resolved in body axes, and $\boldsymbol{\omega}_{b}$ denotes the angular rates of the body frame. The indices $B$ and $E$ refer to rigid body and elastic contributions, respectively. Structural damping can be introduced in terms of modal damping in the $\boldsymbol{D}_{E E}$ submatrix.

\section{Numerical Studies using the X-HALE UAV Configuration}

The method derived in the previous sections was successfully validated statically and dynamically with a slender cantilever beam and with a wingbox made from fiber reinforced material. Furthermore, steady and dynamic aeroelastic simulations have been performed that showed good agreement to nonlinear reference solutions. ${ }^{1,17}$

The X-HALE UAV from University of Michigan is the test case used in this paper to demonstrate and validate steady and unsteady results from the method proposed. The X-HALE is a flexible, high aspect ratio wing-boom-tail type aircraft. It has a wing span of six meters, five booms with horizontal tails attached, and five motor pods with propellers, batteries, and processor boards. The mass of the model is $11 \mathrm{~kg}$ with an 
anticipated flight speed ranging from 10 to $19 \mathrm{~m} / \mathrm{s}^{3}$ The goal of the corresponding activities is to design and build a very flexible UAV for nonlinear aeroelastic tests. ${ }^{18}$ It should serve as a low cost platform to obtain relevant nonlinear aeroelastic data to support validation of nonlinear aeroelastic code and also as future platform for control law studies. Unique coupled nonlinear aeroelasticity/flight dynamics interactions in very flexible aircraft not easily obtainable from wind tunnel tests should be captured. Elaborated on-board measurement systems with compley sensor arrays are collecting data during the flight tests.

Several structural and aerodynamic properties of the aircraft are published in Ref. 3. The wings of the model are fabricated using composite materials, composite beams are used to create a representative simulation model thereof. The reference solutions of the X-HALE test case presented in the following were modeled with University of Michigan's UM/NAST solver. This toolbox provides excellent reference data in terms of nonlinear structural dynamics and coupled aeroelastic simulations. ${ }^{19}$

A 3D finite element model of the X-HALE was build using MSC Nastran. This step is necessary to enable the simulation of the aircraft in the mentioned simulation framework. Any beam-like members (wings, booms, and tails) are modeled by beam-type elements (MSC Nastran PBEAM-elements in this case), discrete mass elements are added to consider concentrated masses of structural members and ballast masses. Only one mass configuration of the model is considered and the mass of the aircraft remains constant during the flight. The structural model is depicted in Fig. 2, where the masses of the discrete mass points are visualized in terms of spheres with a radius equivalent to the density of aluminum. The UM/NAST structural model uses
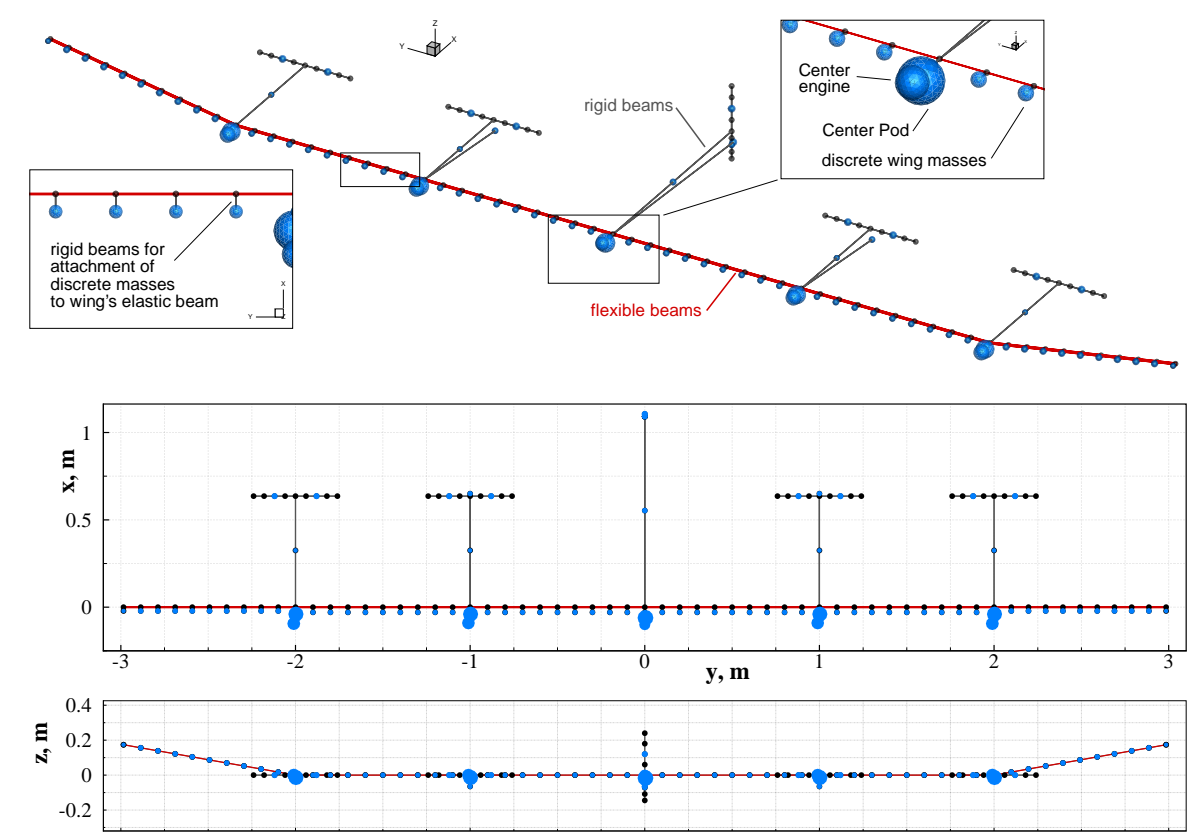

Figure 2. Structural model of the X-HALE UAV build with flexible (red lines) and rigid (grey lines) beam finite elements. Discrete mass points are visualized as spheres with aluminum equivalent density.

various mass offsets to account for different locations of the beam's elastic axis and its mass center of gravity. Since a thorough analysis of how mass offsets behave in Nastran's nonlinear solutions is to be done, these offsets are avoided and "resolved" by additional massless beam elements. These beams are depicted in detail in Fig. 2 at the right wing. It must be mentioned that the structural model is clamped at a node in the symmetry plane (next to the center of gravity) of the aircraft (cf. Fig. 3). The reason for the clamping is that the determination of the higher-order stiffness and mode components requires a set of nonlinear structural simulations which can only be done with a clamped structure.

The lowest twelve natural modes of the FE model are plotted in Fig. 3, the corresponding frequencies are given in Hz. Higher order stiffness and mode components were calculated for these modes and used in any static and dynamic simulations presented in the following sections. Due to the clamping of the model, the modes appear in paires of the right and the left wing. However, the particular modes and corresponding frequencies of the same modes are slightly different due to an unsymmetric setup of the model. The high flexibility of this configuration can be seen in terms of the first bending mode's frequency, which is only about $0.6 \mathrm{~Hz}$. Furthermore, a specific characteristic of the model is that the second mode of each wing is 


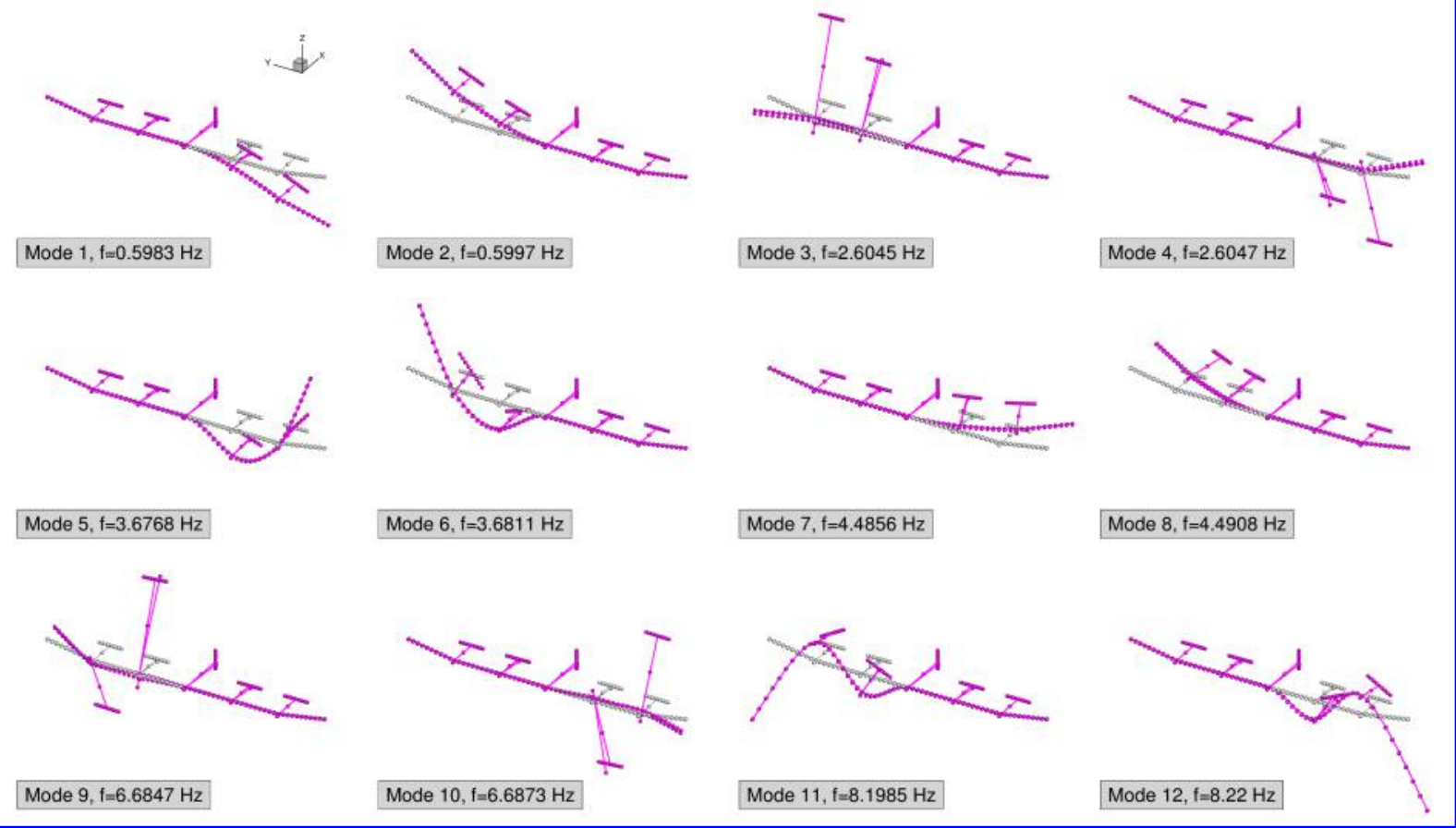

Figure 3. The lowest twelve mode shapes of the X-HALE structural model obtained from a normal modes analysis.

the first torsion mode. Table 1 lists the lowest twelve modes with the corresponding natural frequencies and the types of the modes.

Table 1.
\begin{tabular}{l|l|c|} 
Mode & Type of mode & Eigenvalue FE analysis [Hz] \\
\hline 1 & first bending left & 0.5983 \\
2 & first bending right & 0.5997 \\
3 & first torsion right & 2.6045 \\
4 & first torsion left & 2.6047 \\
5 & second bending left & 3.6768 \\
6 & second bending right & 3.6811 \\
7 & first bending in-plane left & 4.4856 \\
8 & first bending in-plane right & 4.4908 \\
9 & second torsion right & 6.6847 \\
10 & second torsion left & 6.6873 \\
11 & third bending right & 8.1985 \\
12 & third bending left & 8.2201
\end{tabular}

The aerodynamic model consists of different lifting surfaces for the wings, the tails, and the spines. A vortex-lattice method is used to provide the steady and unsteady aerodynamic forces. This method is seen advantageously for the modeling of the X-HALE UAV, since it accounts for the influences of several lifting surfaces among each other and due to the comparatively low computational costs even for unsteady simulations. Furthermore, the aerodynamic panels can undergo any kind of translation and rotation due to e.g. elastic deformations. The VL grid of the X-HALE model is shown in Fig. 4. All elements are lifting surfaces and share wake panels. The real model is equipped with a EMX07 reflex airfoil, the VL solver uses its camber line which is calculated from the upper and lower airfoil coordinates. It must be mentioned that the incidence of the wing's airfoil is five degrees with respect to the body system's reference frame, as can be 

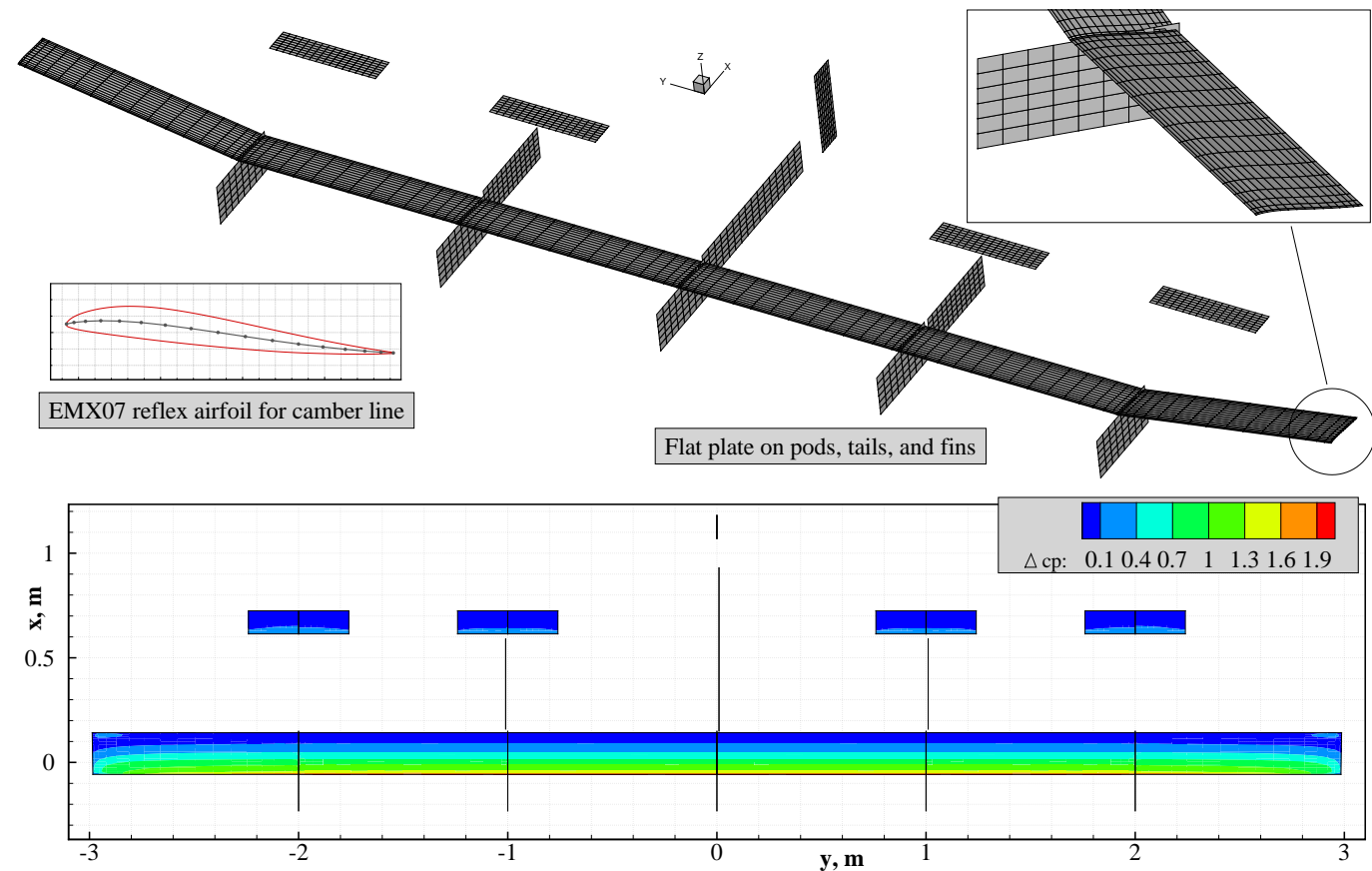

Figure 4. Vortex-lattice grid setup of the X-HALE aerodynamic model composed of several lifting surfaces for the wings, the tails, and the pods. Example of pressure coefficient distribution along the wing is presented.

seen in Fig. 4. A cosine function is applied for the spacing of the panels on the wing in spanwise direction to better resolve the wing tip effect, the transition at the dihedral wing elements and at the pods. Any other lifting surfaces are modeled as flat plates. An example of the pressure coefficient distribution on the wing is shown in the same plot. As can be seen, three-dimensional aerodynamic effects are pronounced at the wing tips.

A static polar was calculated (using the rigid VL model) for a flow velocity of $16 \mathrm{~m} / \mathrm{s}$ and a fluid density of $1.2216 \mathrm{~kg} / \mathrm{m}^{3}$. The angle of attack (AoA) was varied between -3 and 3 degrees in steps of one degree. The forces in the $x$ and in the $z$ directions are presented together with the moment around the $y$ axis in Fig. 5. Additionally, the force and moment coefficients are given in the same plot. As can be seen, a lift
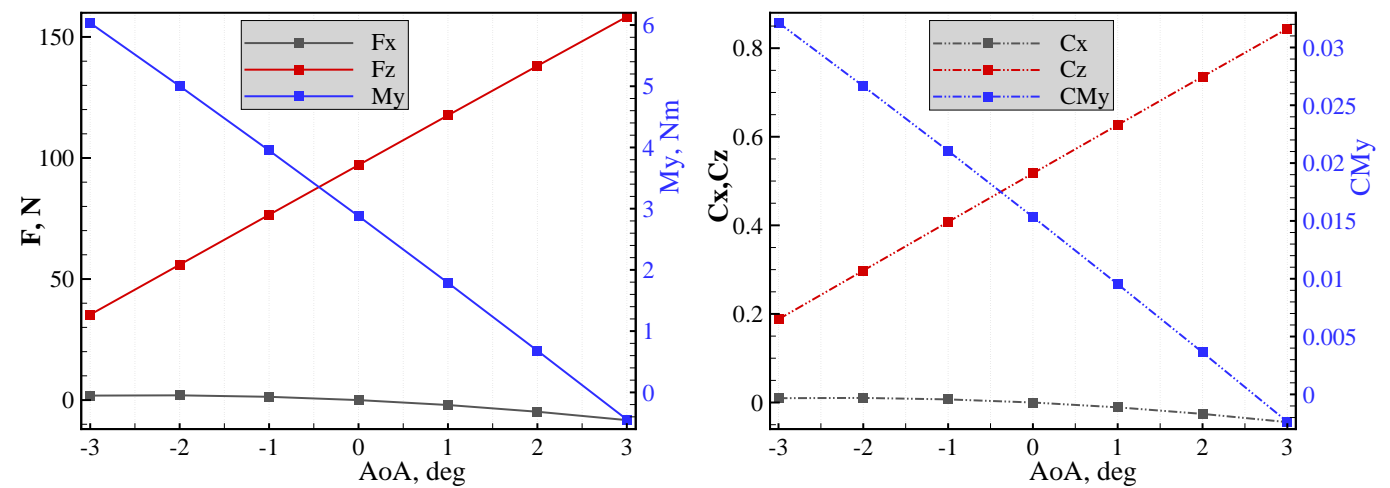

Figure 5. Drag force, lift force, and pitching moment of the rigid X-HALE model as function of AoA with corresponding coefficients in the body fixed frame. Velocity $=16 \mathrm{~m} / \mathrm{s}$, fluid density $=\mathbf{1 . 2 2 1 6} \mathrm{kg} / \mathbf{m}^{3}$.

force equivalent to the weight of the aircraft can be reached at an angle of attack of the body fixed frame of about 0.5 degrees. As mentioned above, the incidence of the wing's airfoil based on the body frame is five degrees, thus a low body angle of attack is needed to balance the weight of the aircraft. The lift force in the $z$ direction normalized per length for these angles of attack is shown in Fig. 6. The linear characteristics of the lift force with respect to the angle of attack is obvious. Being a linear aerodynamic method based on potential flow, the vortex lattice method is based on a linear relationship between the circulation on the 


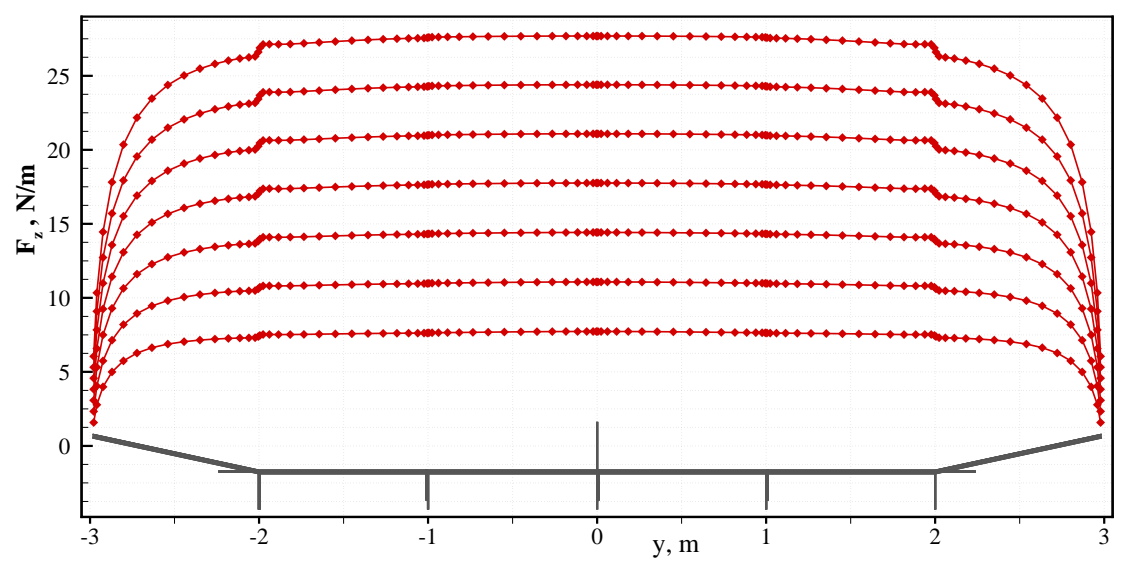

Figure 6. Lift force per length in the $z$ direction for different angles of attack (-3 degrees to 3 degrees). Velocity $=$ $16 \mathrm{~m} / \mathrm{s}$, fluid density $=1.2216 \mathrm{~kg} / \mathrm{m}^{3}$.

wing's panels and the corresponding kinematic boundary condition. Since no stall effects are modeled in this implementation, the lift of a particular panel is always a linear function of the panel's circulation.

Coupled aeroelastic solutions require the transformation of aerodynamic forces onto the structural nodes and the interpolation of structural deformations onto the aerodynamic grid. The force transfer is done via the transposed of a coupling matrix. ${ }^{20}$ However, transforming aerodynamic forces into equivalent forces on the beam's nodes requires the calculation of forces and moments. In this case, another approach was used to avoid the calculation of moments. A so-called coupling model was built which uses rigid-bar elements that are connected to a beam node at one side each. The other end of the rigid-bar elements are used as coupling points to where the aerodynamic forces are transferred by the coupling matrix. ${ }^{1}$ The structural model is therefore enhanced by massless rigid bars, as shown in Fig. 7.

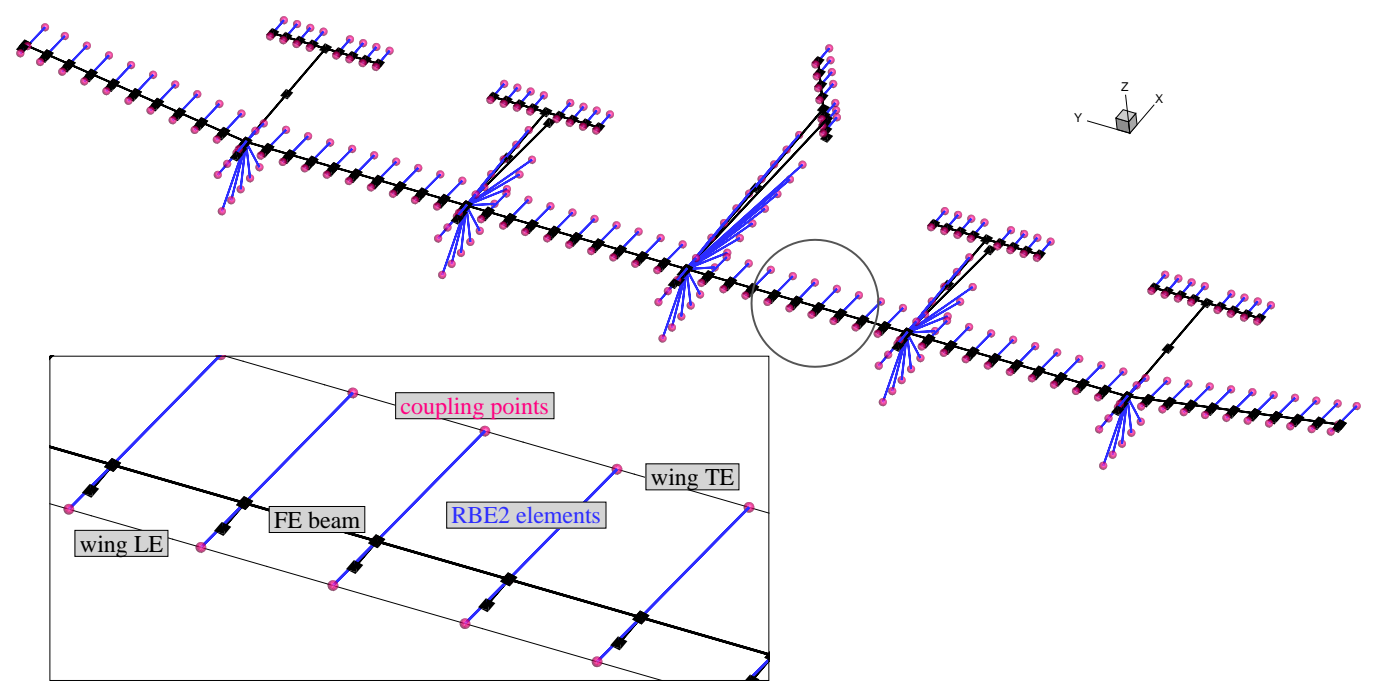

Figure 7. Coupling model of the X-HALE UAV. Nastran's RBE2 elements (massless and rigid bars) are used to introduce the aerodynamic forces into the beam's nodes.

\section{Static response of the structural model}

Two kinds of static simulations will be presented to verify the higher order stiffness and mode components that were calculated for the FE model. First, a constant tip load in the $z$ direction which is applied at the outermost node of the wings's dihedral segment. Second, a constant loading, also in $z$ direction, which is applied at every node of the wing's flexible beam nodes. No gravity and no follower forces are considered. 
The values of the forces are selected to obtain maximum deformations in the $z$ direction of more than $35 \%$ half span of the model (three meters). Results from a fully nonlinear Nastran solution (solution sequence 400) are compared to results from the proposed method. The result of the first and the second simulation

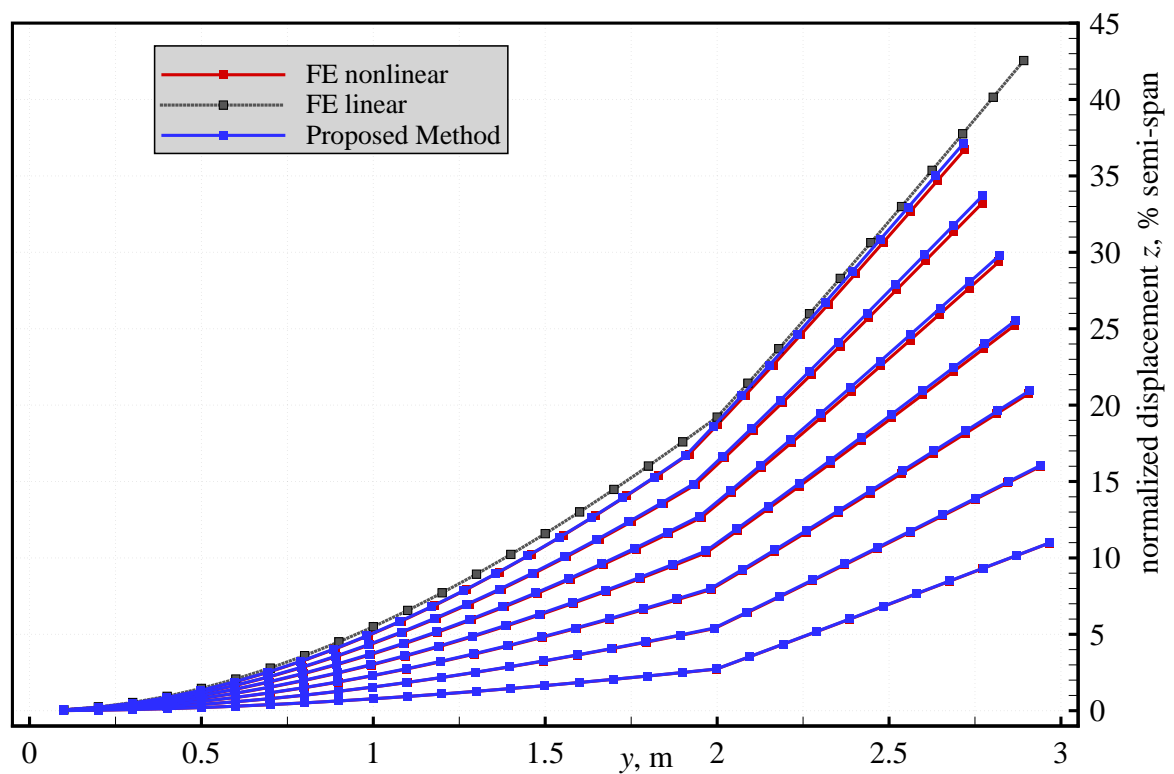

Figure 8. Comparison of the static displacement field obtained by different methods for forces from $2 N$ to $14 N$ (in steps of $2 \mathrm{~N}$ ) applied along the $z$ direction at the end of the wing.

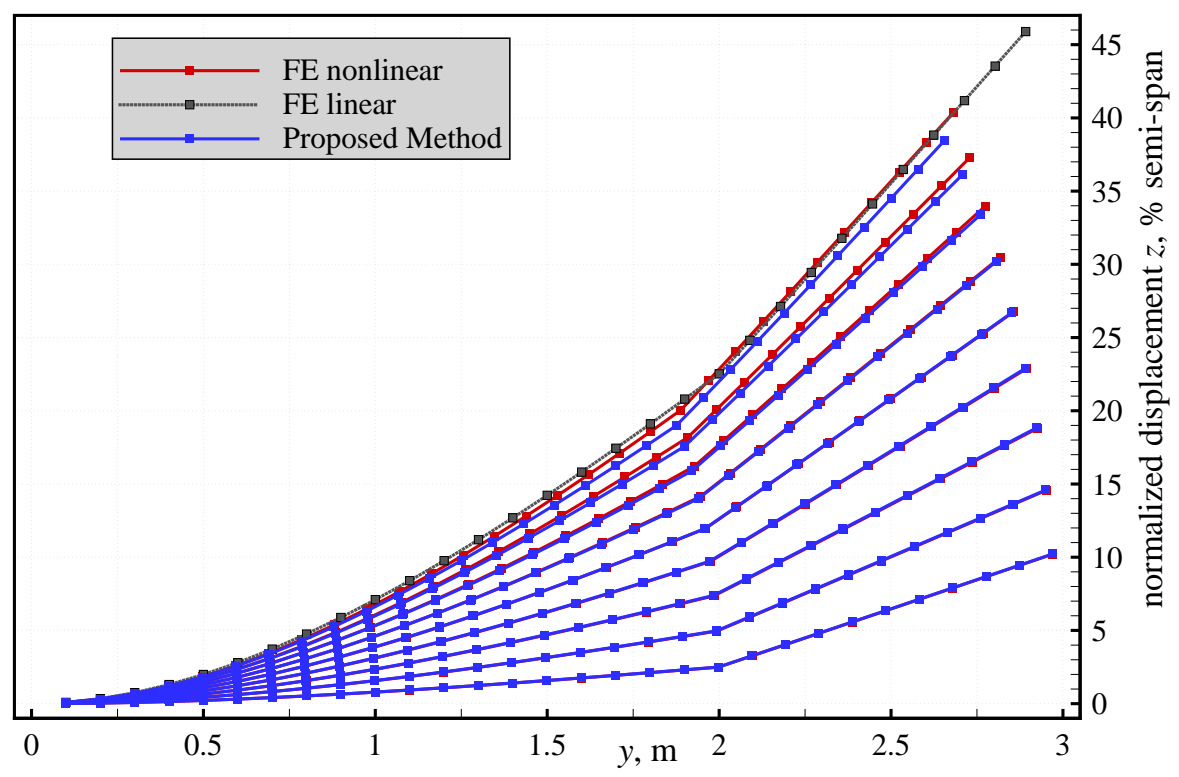

Figure 9. Comparison of the static displacement field obtained by different methods for forces from $0.2 N$ to $1.8 N$ (in steps of $0.2 \mathrm{~N}$ ) applied along the $z$ direction at every node of the wing.

are shown in Fig. 8 and Fig. 9, respectively. As can be seen, a good agreement between the nonlinear reference solution and the enhanced modal approach is given up to a maximum tip displacement of about $30 \%$ half span. For reference, the solution of a linear FE simulation is added for the highest force field but shows large deviations to the nonlinear results. 


\section{Steady trim simulations for the rigid and the elastic aircraft}

In the following, results of steady trim simulations are presented. Therefore, the flight velocity was varied within $15,16,17$, and $18 \mathrm{~m} / \mathrm{s}$. The density of the fluid was set to $1.2216 \mathrm{~kg} / \mathrm{m}^{3}$. Trimming the aircraft is done by fulfilling force and moment balance in the aircraft's body fixed system. In this case, three parameters are used to achieve a steady state horizontal flight. The angle of attack, the angle of the tail planes, and the thrust of the engines.

For the first trim simulation, the flexibility of the aircraft was set to zero and thus no elastic deformations were taken into account. The reason for this approach is that possible differences in the results of UM/NAST and the VL solver can be revealed easier. The VL solver uses an iterative procedure based on the NewtonRaphson method to converge a residual (the resulting forces and moments in the body fixed system) to zero. Therefore, a Jacobian matrix is calculated which contains the partial derivatives of the residuals with respect to the trim parameters. The UM/NAST and the VL solver results of the rigid trim simulations as function of the trim velocity are shown in Fig. 10. The tail rotation angle is the same for all four outer tails, a positive tail rotation angle means that the tail produces a more negative pitching moment. In general, the angle of

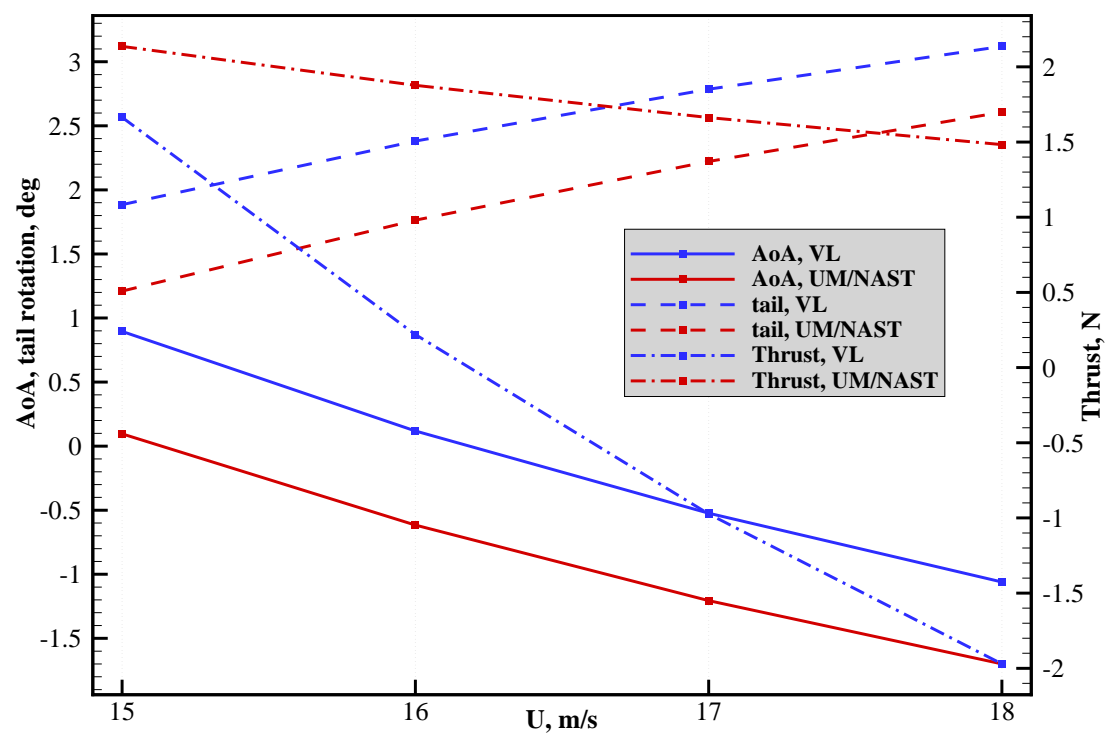

Figure 10. Results of the static trim simulations of two methods of the rigid X-HALE model for flight velocities in the range of $15 \mathrm{~m} / \mathrm{s}, 16 \mathrm{~m} / \mathrm{s}, 17 \mathrm{~m} / \mathrm{s}$, and $18 \mathrm{~m} / \mathrm{s}$.

attack and tail rotation results show similar trends between the two methods. However, the difference of the magnitudes of the AoA is about 0.8 degrees, the difference in the tail rotation angle is about 0.7 degrees. This can be explained with the fact that UM/NAST calculates higher aerodynamic forces along the wingspan and thus requires a lower angle of attack. The tail rotation angles of both methods are increasing (meaning the tails are producing higher negative pitching moments) with increasing flight velocity. This behaviour is rather unusual, and can be attributed to the increased positive pitching moment the EMX07 reflex airfoil produces at higher flow velocities. The thrust shows large deviations between both methods. UM/NAST requires a thrust of about $2 \mathrm{~N}$ for flying at $15 \mathrm{~m} / \mathrm{s}$; this value is reduced evenly to $1.5 \mathrm{~N}$ at $18 \mathrm{~m} / \mathrm{s}$. The reason therefore is the reduction of the induced drag due to the reduced lift coefficient required at higher flight speeds. However, the VL solver yields a negative thrust at flight speeds starting at $16 \mathrm{~m} / \mathrm{s}$. The reason is that in the VL solver, the thrust calculated in the trim process is the force that is required to balance the resulting aerodynamic and inertial forces in the $x$ direction in the body frame. It must be mentioned that the $x$ axis of the body frame in the VL solver points from tail to nose, and a negative value for the thrust means that this is a "positive" thrust force in its basic sense. Furthermore, the solver only calculates the induced drag due to lift in the body frame. Thus, at the positive angle of attack (cf. flight speed of $15 \mathrm{~m} / \mathrm{s}$ ), the gravity and the drag force both have a positive component in the body frame's $x$ direction which must be cancelled with a negative force of equal magnitude.

Since the higher order mode components are calculated by a series of nonlinear simulations, where the FE model is clamped at a certain structural node, the elastic trim process requires the consideration of inertial 
forces. Here, only the gravity forces at each mass point are taken into account since the aircraft is trimmed to a steady horizontal flight where both body frame angular and translational rates and accelerations are equal to zero. If a linear FE model with free-free mode shapes would be used for trim, no inertial loads had to be considered at the FE nodes since inertial loads do not contribute to structural deformations in this case (they only contribute to rigid-body motions).

The results of the elastic trim process in terms of the same parameters as for the rigid one are presented in Fig. 11. A similar trend as for the rigid trim results can be seen in the plot. However, the magnitudes

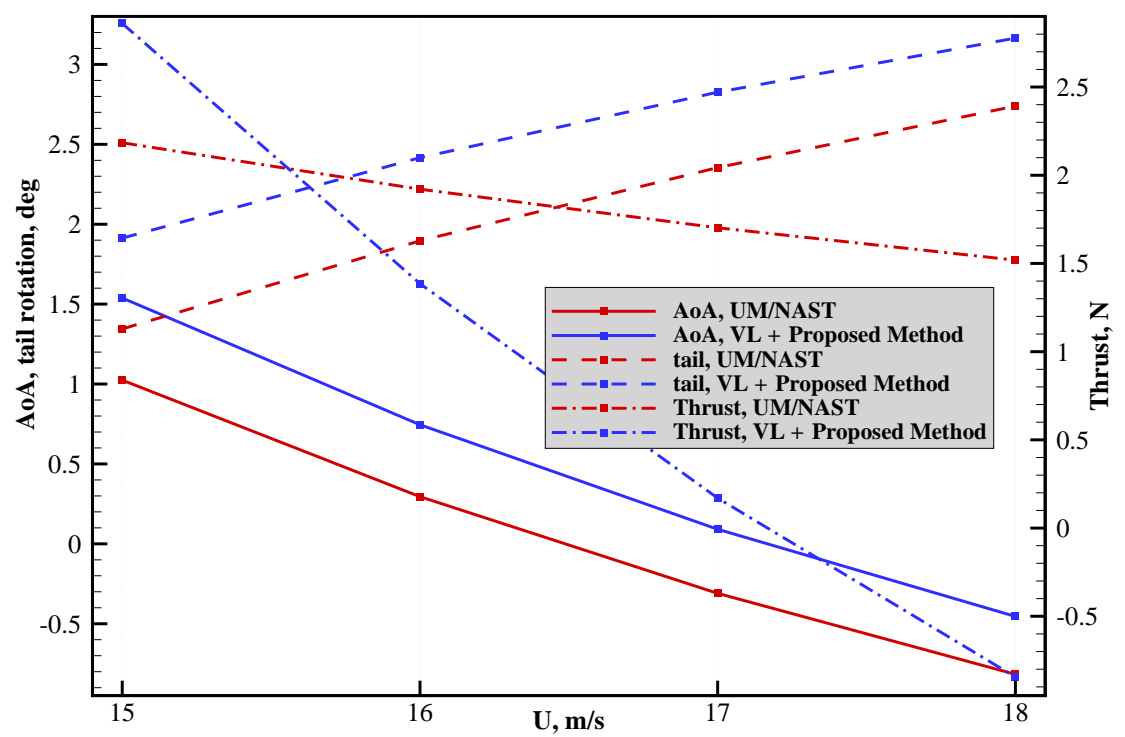

Figure 11. Results of the static trim simulations of two methods of the elastic X-HALE model for flight velocities in the range of $15 \mathrm{~m} / \mathrm{s}, 16 \mathrm{~m} / \mathrm{s}, 17 \mathrm{~m} / \mathrm{s}$, and $18 \mathrm{~m} / \mathrm{s}$.

of the angle of attack are increased compared to the rigid results. Due to the flexible deformation of the wing, a part of the aerodynamic force of the wing has a component in the $y$ direction. Thus, depending on the amount of flexible deformation, the angle of attack must be increased. The tail angles and the thrust are slightly higher in the elastic case. UM/NAST yields roughly the same thrust as in the rigid trim case, whereas the thrust of the VL solver result is less negative in the elastic trim case.

Comparing the elastic deformations calculated by the two different methods shows that the VL solver yields higher overall displacements than UM/NAST. Fig. 12 depicts the elastic deformation of the left and the right wing obtained from UM/NAST and the VL method for a trim speed of $16 \mathrm{~m} / \mathrm{s}$. The VL method

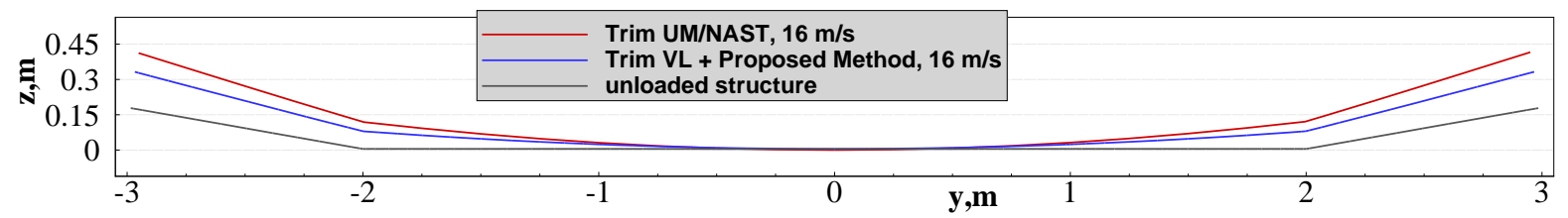

Figure 12. Structural deformation of the X-HALE left and right wing obtained by two methods for trim velocity of $16 \mathrm{~m} / \mathrm{s}$.

uses the proposed method for the calculation of the structural displacements. Additionally, the undeformed structure is shown in this figure for reference. Due to an equal distribution of masses along the wings (pods, engines, tails), the overall structural deformations of the model are comparatively small. In the trimming process, both methods must yield the same force in the $z$ direction to balance the weight of the aircraft. Thus differences in the resulting structural deformation are most likely to come from different lift distributions over the wing. Due to the high flexibility of the wing, small variations of the lift forces lead to high variations in the structural deformations. Differences between the two methods in terms of structural deformations are much larger than the differences due to the range of trim velocities considered $(15,16,17$, and $18 \mathrm{~m} / \mathrm{s})$, as can be seen in Fig. 13 . 


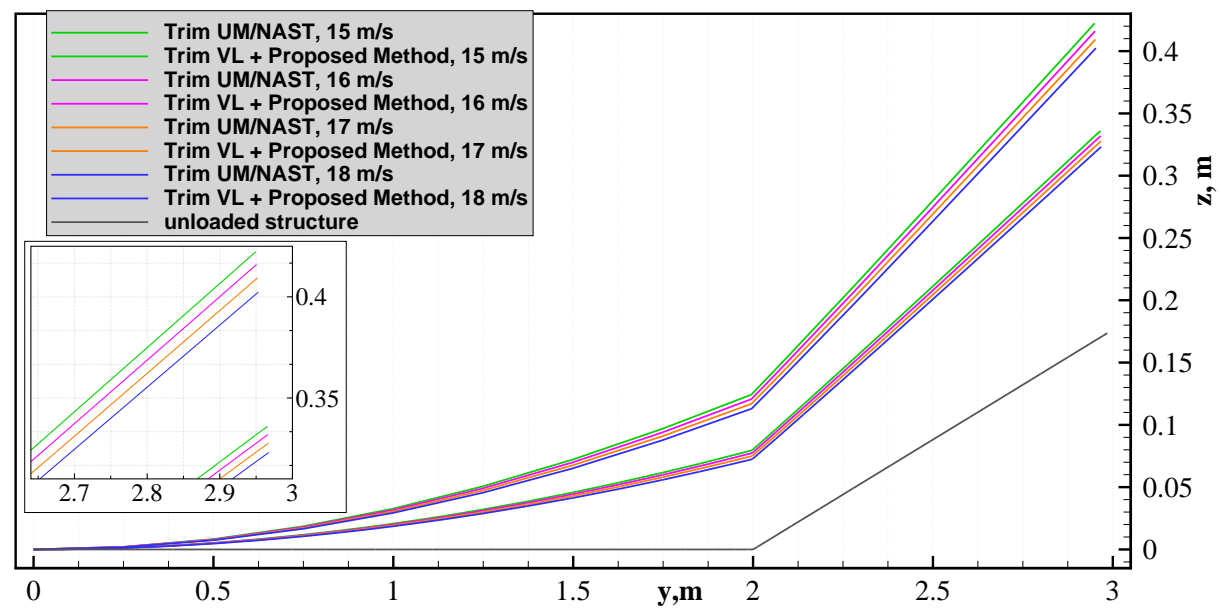

Figure 13. Differences in structural deformations of the X-HALE right wing in detail obtained by two methods for trim velocities in the range of $15,16,17$, and $18 \mathrm{~m} / \mathrm{s}$.

\section{Unsteady simulations}

Unsteady results are presented in the following for two sinusoidal tail input scenarios. The unsteady version of the VL solver, named UVL in the following, uses a wake-stepping method to account for the unsteady circulation on body and wake panels. ${ }^{21}$ An implicit $B D F$ scheme was used to solve the coupled equations (Eq. ??) in time with a timestepsize of $0.05 \mathrm{~s}$. Since the governing equations describe an initial value problem, a steady trim simulation was performed for a flight velocity of $16 \mathrm{~m} / \mathrm{s}$ and the results thereof used as initial conditions for the unsteady simulations. This approach also ensures that no rigid body or elastic motions are excited within the first time steps of the unsteady simulations, which could possibly lead to smal drifts. In contrast to e.g. aeroelastic simulations based on a frequency doman solution of the doublet lattice method, time accurate solutions require converged steady initial solutions.

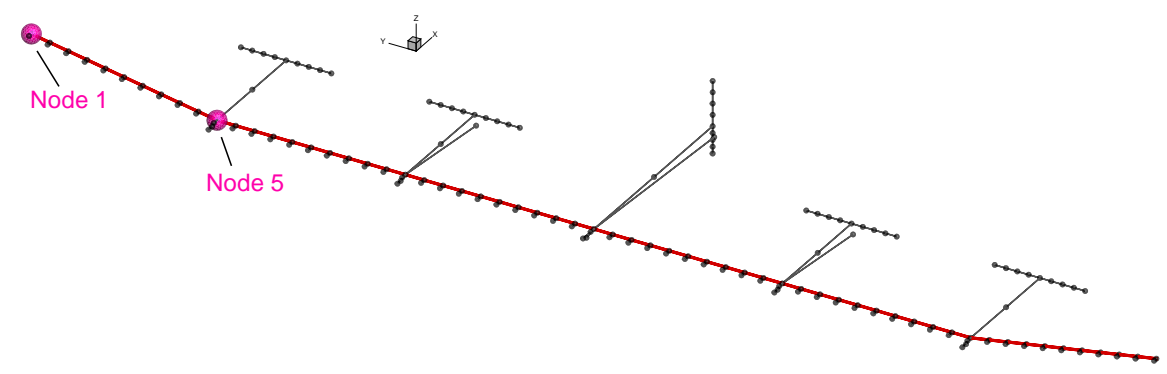

Figure 14. Structural Nodes 1 and 5, for which time dependent displacements will be presented.

The results of the unsteady simulation are presented in terms of the displacement of two structural nodes located on the right wing's beam reference line at $y=2 m$, and $y=3 m$, cf. Fig. 14 .

Results of a sinusoidal rotation of the four outer tails of the X-HALE model are presented within a time interval from 0 to 14 seconds. The first amplitude of the rotation is 0.25 degrees, the period of rotation is 4 seconds. This amplitude is comparatively small and was chosen to obtain results within the linear regime of structural deformations and small rigid body motions. Structural deformations are plotted in Fig. 15 for the two nodes mentioned. The corresponding rigid body motion as function of time is presented in Fig. 16 in terms of the location in the $z$ direction of the body frame's origin in a geodetic system, its time derivative (velocity of the body frame in the $z$ direction, resolved in the geodetic system), and the angle of pitch. As can be seen from the results, very small structural deflections are excited by the tail rotation manoeuvre. Though very small in the magnitude, large differences between the UM/NAST and the UVL solution with the proposed method exist. The UM/NAST solution yields smooth structural deflections with a much lower amplitude and a more or less constant oscillation compared to the UVL results for both nodes. Slight higher 


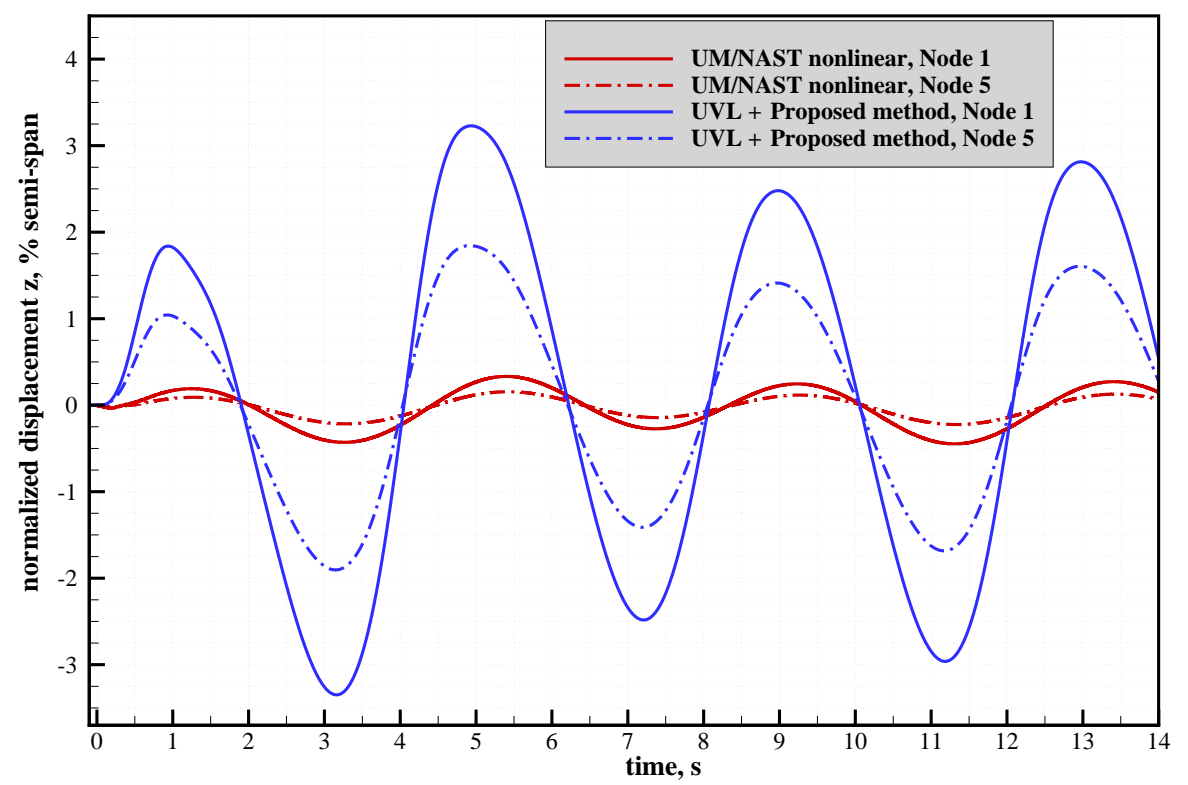

Figure 15. Normalized displacements of keypoints 1 and 5 as function of time for a continuous sinusoidal tail input with an amplitude of 0.25 degrees and a period of 4 seconds. Steady trim velocity is $16 \mathrm{~m} / \mathrm{s}$.
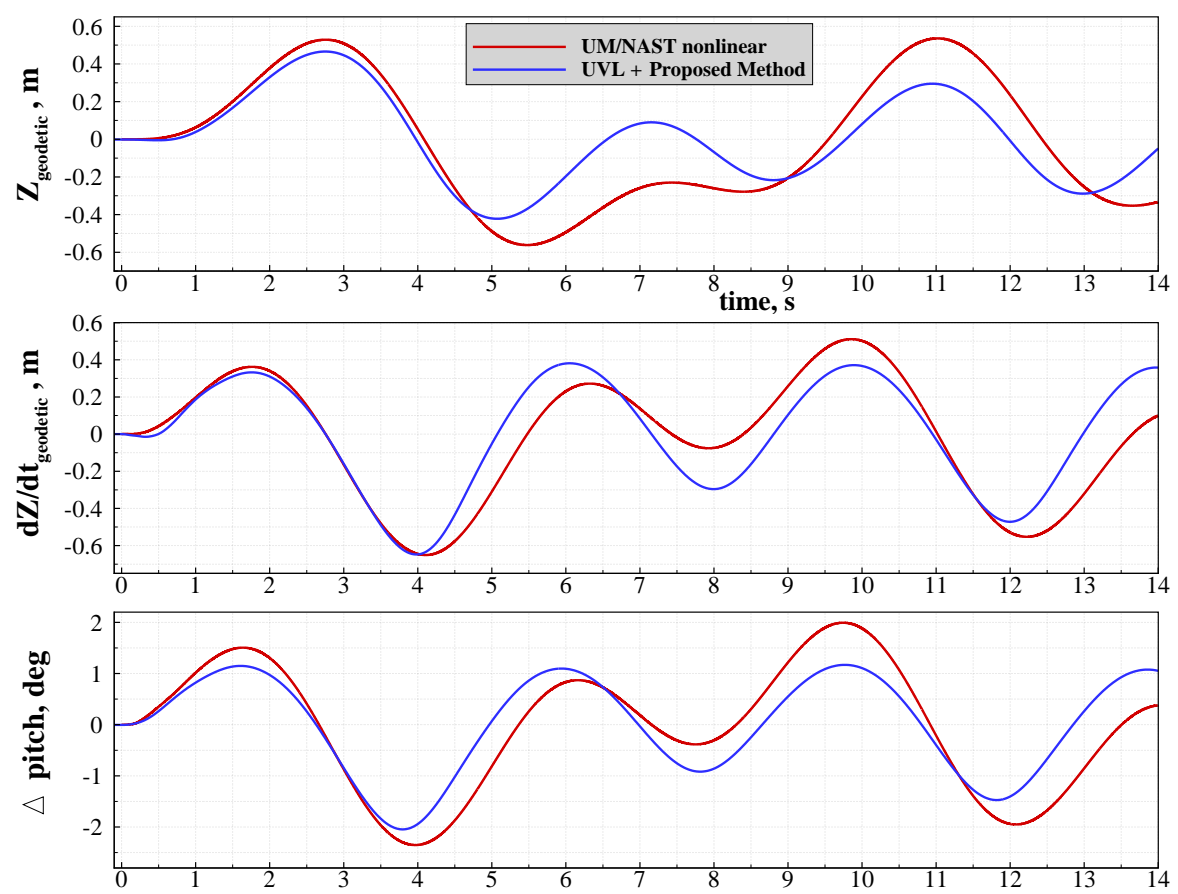

Figure 16. Position and velocity of the X-HALE's body frame in the geodetic system, and pitch angle as function of time for a continuous sinusoidal tail input with an amplitude of 0.25 degrees and a period of 4 seconds. Steady trim velocity is $16 \mathrm{~m} / \mathrm{s}$.

frequency oscillations are present in the UVL solver results with pronounced peaks in the region of about 1 and 5 seconds. The rigid body results are more comparable in the trend. It should be mentioned that the rigid body motion agrees well for both methods within the first four seconds of the simulations.

The second unsteady manoeuvre uses the same type of excitation, but this time the amplitude of the tail rotation is twice as high. All other simulation parameters are kept the same. Nodal displacements are shown in Fig. 17. Due to the larger amplitude, significantly higher structural deformations are excited, up to $15 \%$ tip displacement in the UVL solver solution. Again, the UM/NAST solution shows a smooth oscillation for 


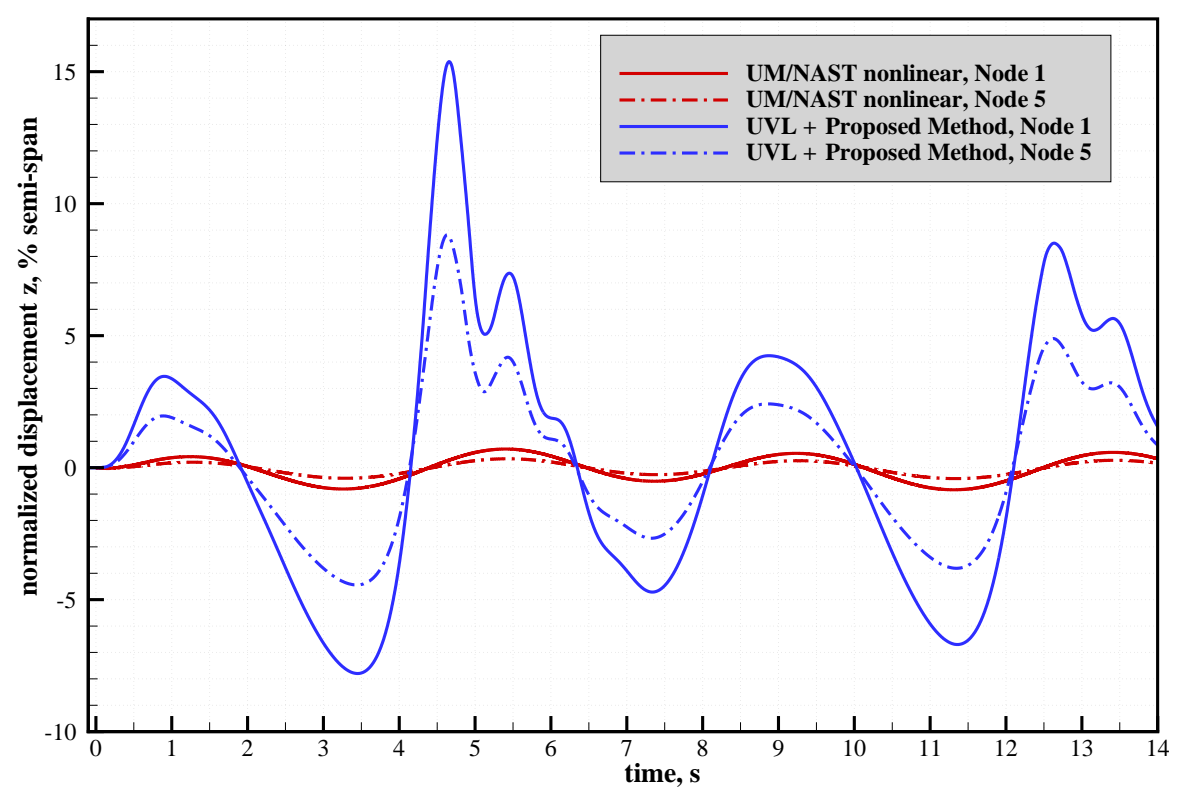

Figure 17. Normalized displacements of keypoints 1 and 5 as function of time for a continuous sinusoidal tail input with an amplitude of 0.5 degrees and a period of 4 seconds. Steady trim velocity is $16 \mathrm{~m} / \mathrm{s}$.
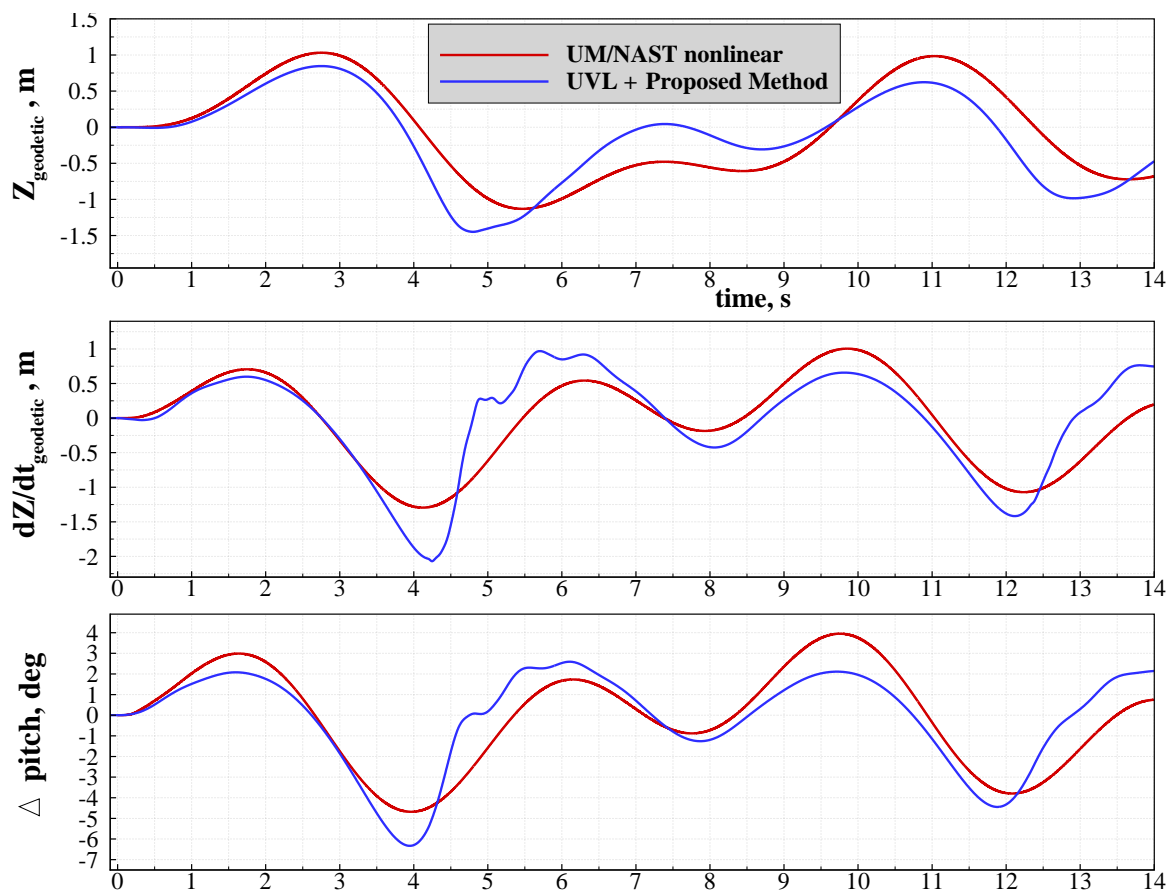

Figure 18. Position and velocity of the X-HALE's body frame in the geodetic system, and pitch angle as function of time for a continuous sinusoidal tail input with an amplitude of 0.5 degrees and a period of 4 seconds. Steady trim velocity is $16 \mathrm{~m} / \mathrm{s}$.

both nodes with almost constant amplitude over the time interval considered. This time, the UVL solver results show displacements with distinctive contributions of modes with higher frequency. A similar trend can be seen in terms of the rigid body motion for this test case, as shown in Fig. 18.

As can be expected, the amplitudes of the location and the velocity of the body system's frame of reference, as well as the pitch angle are almost twice as high as for the first test case. Good agreement between the results of the two solution methods is given for the first three seconds of simulation, but results drift apart shortly after. In general, the agreement of the unsteady manoeuvre results between the 
fully nonlinear solution from UM/NAST and the UVL solver with the method proposed is not as good as expected. It seems that the structural flexibility of the UM/NAST aeroelastic model is much higher than the model which is used with the UVL solver. The clarification of the differences in the unsteady results will be the next activities of the X-HALE model aeroelastic modeling.

\section{Conclusion and Outlook}

A method was presented that enhances the classical modal approach used in structural dynamics and aeroelastic applications towards large geometric deformations. Nonlinearities in the force-displacement relationships and in the geometrically nonlinear deformation field are accounted for. Therefore, the classical modal approach governing equations are enhanced with terms of higher order for the stiffness and the reconstruction of the displacement field in physical coordinates from modal space. Based on these terms, nonlinear static and dynamic governing equations were obtained in which the generalized stiffnesses are depending on the forces applied to the structure. It was shown that the quadratic component of the higher order modes are needed to account for in-plane loadings, which are present in typical aerodynamic forces when lifting panels undergo large rotations. In this work, the method was applied to the X-HALE UAV model from University of Michigan. Results of static response of the structural model and steady trim simulations were presented. The static response shows good agreement to full nonlinear reference solutions up to about $30 \%$ wing tip displacement. The trim simulations reveal smaller differences between the UM/NAST and the VL based results. However, these differences are most likely to come from different approaches to the modeling of aerodynamic forces. Unfortunately, only poor agreement is given for the two unsteady manoeuvre simulations between UM/NAST and the UVL solver with the proposed method. These differences will be analyzed and can hopefully be eliminated. Topics of future research with the X-HALE model and the proposed method are further simulations of different kinds of unsteady manoeuvres, such as time-dependent deflections of control surfaces and gust encounter.

\section{Acknowledgments}

Parts of this work were carried out during two internships of the first author at the Active Aeroelasticity and Structures Research Lab at the University of Michigan. The authors would like to thank the entire team of the laboratory for their support. Furthermore, the support of the DLR Institute of Aeroelasticity for this research topic is gratefully acknowledged.

\section{References}

${ }^{1}$ Ritter, M., Cesnik, C. E. S., and Krüger, W. R., An Enhanced Modal Approach for Large Deformation Modeling of Wing-Like Structures, AIAA SciTech, $56^{\text {th }}$ AIAA/ASME/ASCE/AHS/ASC Structures, Structural Dynamics, and Materials Conference, Kissimmee, Florida, Jan 2015.

${ }^{2}$ Cesnik, C. E. S., Palacios, R., and Reichenbach, E. Y., "Reexamined Structural Design Procedures for Very Flexible Aircraft," Journal of Aircraft, Vol. 51, No. 5, 2014, pp. 1580-1591.

${ }^{3}$ Cesnik, C. E. S., Senatore, P., Su, W., Atkins, E., Shearer, C., and Pitchter, N., X-HALE: A Very Flexible UAV for Nonlinear Aeroelastic Tests, Structures, Structural Dynamics, and Materials and Co-located Conferences, $51^{s t}$ AIAA/ASME/ASCE/AHS/ASC Structures, Structural Dynamics, and Materials Conference, Orlando, Florida, Apr 2010.

${ }^{4}$ Mignolet, M. P., Przekop, A., Rizzi, S. A., and Spottswood, S. M., "A review of indirect/non-intrusive reduced order modeling of nonlinear geometric structures," Journal of Sound and Vibration, Vol. 332, No. 10, 2013, pp. 2437 - 2460.

${ }^{5}$ Kim, K., Radu, A. G., Wang, X. Q., and Mignolet, M. P., "Nonlinear reduced order modeling of isotropic and functionally graded plates," International Journal of Non-Linear Mechanics, Vol. 49, No. 0, 2013, pp. 100 - 110.

${ }^{6}$ Wang, X. Q., Perez, R. A., and Mignolet, M. P., "Nonlinear Reduced Order Modeling of Complex Wing Models," $54^{t h}$ AIAA/ASME/ASCE/AHS/ASC Structures, Structural Dynamics, and Materials Conference, Boston, Massachusetts, Apr 2013.

${ }^{7}$ Kuether, R. J. and Allen, M. S., "Nonlinear Modal Substructuring of Systems with Geometric Nonlinearities," Structures, Structural Dynamics, and Materials and Co-located Conferences, $54^{t h}$ AIAA/ASME/ASCE/AHS/ASC Structures, Structural Dynamics, and Materials Conference, Boston, Massachusetts, Apr 2013.

${ }^{8}$ Kuether, R. J. and Allen, M. S., "Substructuring with Nonlinear Reduced Order Models and Interface Reduction with Characteristic Constraint Modes," AIAA SciTech, $55^{\text {th }}$ AIAA/ASME/ASCE/AHS/ASC Structures, Structural Dynamics, and Materials Conference, National Harbor, Maryland, Jan 2014.

${ }^{9}$ Segalman, D. J. and Dohrmann, C. R., "Dynamics of Rotating flexible Structures by a Method of Quadratic Modes," Sandia National Laboratories, Structural Dynamics Division, Albuquerque, New Mexico, 1990.

${ }^{10}$ Segalman, D. J. and Dohrmann, C. R., "A Method for Calculating the Dynamics of Rotating Flexible Structures, Part1: Derivation," ASME Journal of Vibration and Acoustics, Vol. 118, 1996, pp. 313-317. 
${ }^{11}$ van Zyl, L. H., Sutherland, A. N., and Rossouw, P. S., "Parabolic Mode Shapes: What they are, where to get them and what to do with them," International Forum on Aeroelasticity and Structural Dynamics, Seattle, Washington, 2009.

12 van Zyl, L. H. and Mathews, E. H., "Quadratic Mode Shape Components From Linear Finite Element Analysis," ASME Journal of Vibration and Acoustics, Vol. 134, Feb. 2012

${ }^{13}$ Shames, I. H. and Dym, C. L., Energy and Finite Element Methods in Structural Mechanics, Hemisphere Publishing Corporation, 1985.

14 MEIROVITCH, L., "Hybrid state equations of motion for flexible bodies in terms of quasi-coordinates," Journal of Guidance, Control, and Dynamics, Vol. 14, No. 5, Sep 1991, pp. 1008-1013.

${ }^{15}$ BUTTRILL, C., ARBUCKLE, P., and ZEILER, T., Nonlinear simulation of a flexible aircraft in maneuvering flight, Guidance, Navigation, and Control and Co-located Conferences, American Institute of Aeronautics and Astronautics, Aug 1987.

${ }^{16}$ Brockhaus, R., Flugregelung, Springer Berlin Heidelberg, 2013.

17Ritter, M. and Cesnik, C. E. S., Large Deformation Modeling of a Beam Type Structure and a 3D Wingbox using an Enhanced Modal Approach, AIAA SciTech, $57^{\text {th }}$ AIAA/ASME/ASCE/AHS/ASC Structures, Structural Dynamics, and Materials Conference, San Diego, California, Jan 2016.

18 "Information about the X-HALE UAV," http://gust.engin.umich.edu/research/xhale.html, June 2015.

${ }^{19} \mathrm{Su}$, W. and Cesnik, C. E. S., "Nonlinear Aeroelasticity of a Very Flexible Blended-Wing-Body Aircraft," Journal of Aircraft, Vol. 47, No. 5, Sep 2010, pp. 1539-1553.

${ }^{20}$ Anon., MSC Nastran 2012 Quick Reference Guide, MacNeal-Schwendler Corporation, 2011.

${ }^{21}$ Katz, J. and Plotkin, A., Low-Speed Aerodynamics, Cambridge Aerospace Series, Cambridge University Press, 2001. 